\title{
The Application of Multi-modal Discourse in English Translation of Tujia Folk Song Long Chuan Diao in Western Hubei Province
}

\author{
Hong $\mathrm{Xu}^{\mathrm{a}}$ \\ Foreign Language Department, Hubei University for Nationalities, Enshi, Hubei, France
}

\begin{abstract}
Nowadays, multi-modal discourse, including language, picture, audio and video play a very important role in the translation of folk songs. Under the comprehensive framework of Delu Zhang, this paper analyses the application of multi-modal discourse in the translation of Tujia folk song Long Chuan Diao from four aspects: cultural level, context level, content level and expressive level. The study can better understand Tujia folk songs and transmit Tujia culture. Multi-modal discourse analysis plays an important role in translating and spreading Tujia folk songs in Western Hubei province.
\end{abstract}

\section{Introduction}

In an era of informational age, when multimedia technology develops quickly, there emerges many new media composed of not only linguistic text but also visual images such as music, photos, pictures, videos, and many others. People have found that it is no longer possible to analyse and study discourse from the perspective of language only, because people think that it is not only the image of language but also colour and action symbols participate in the construction of meaning together with linguistic symbols. Thus, multimodal discourse research is in full swing, and the study of multi-modal discourse is strongly interdisciplinary, relying on a rich, varied array of verbal and non-verbal communicative cues, drawing on research ranging from the basic linguistics into the field of psychology, sociology, anthropology, philosophy as well as translation. On the whole, the study of multi-modal translation in China starts relatively late and is not a very mature research field. Most of its analytical theories are applied in film subtitle translation, but few studies have been applied in folk song translation. The traditional translation of folk songs mainly focuses on the analysis of the text level. However, different from other discourses, the folk songs are the combination of singing and performing, audio-visual tactile, picture, language and sound. Taking Long Chuan Diao as an example, this paper discusses the application of multi-modal discourse in translating Tujia folk songs into English, in order to better understand Tujia folk songs and transfer Tujia culture in western Hubei province.

\section{Multi-modal discourse}

Discourse analysis has already achieved a lot during these sixty years since it came into being in 1952. Harris published a paper named discourse analysis and announced the beginning of the research of discourse analysis, during these years discourse analysis has have already developed into an independent discipline itself and many branches. In an informational age, the work of Kress and van Leeuwen is becoming an increasingly definitive approach to analyse text, and the discourse analytical term "multi-modal" originated. Mode (Modality) refers to communicational channels and media, including language, image, colour, music and other symbolic systems. Multi-modal discourse is a phenomenon of communication with each other through language, pictures, sound and other means and symbol resources, involving many senses such as vision, hearing, touch. Kress and Leeuwen has established a multi-modal discourse analysis framework for image analysis based on Halliday's functional grammar theory from the perspective of social semiotics, analysing the functions of images, sounds and colours in discourse. ${ }^{[1]}$ Since then, there has been an upsurge in the study of multi-modal discourse. Multi-modal discourse analysis breaks through the limitations of discourse analysis studies, which focus only on the language system, semantic structure and their relationship with social culture and psychological cognition, and makes people pay attention to images, sounds, colour, and other expressions of meaning in the analysis.

In contrast, the domestic research started late. It does not open the door to the study of multi-modal discourse in China until Professor Zhanzi Li introduced the framework of multi-modal discourse analysis based on systemic functional linguistics. ${ }^{[2]}$ Delu Zhang tries to establish a comprehensive framework of multi-modal discourse analysis according to the theory of systemic functional linguistics, while the framework consists of four levels: (1) cultural level; (2) context level; (3) content level; (4) expressive level. ${ }^{[3]}$ Multi-modal analysis has considered pictures, images and other social symbol systems beyond linguistic barriers to semiotics,

* Corresponding author: ${ }^{\mathrm{a}}$ Hong Xu: 258106276@qq.com 
philosophy, sociology, anthropology, politics, journalism, psychology, law, aesthetics and medicine. In recent years, domestic scholars have explored multi-modal discourse analysis from different perspectives. For example, Dezheng Feng introduced the important role and function of digital technology in multi-modal discourse analysis. ${ }^{[4]}$ Dan Cai thinks that as an important means to realize the cultural communication of ethnic minorities, the minority TV cultural program is a dynamic multimodal discourse, which can be examined from three aspects: the field, the tenor and the style. ${ }^{[5]}$ Yanzhi Liang explores the subtitle translation of British and American movies and TV dramas from the perspective of multimodal discourse analysis. ${ }^{[6]}$ Weiping Zhao demonstrates how to establish a theoretical framework of multi-modal discourse analysis in the flipping classroom, and analyses the feasibility of using multi-modal discourse analysis theory to study the flipping classroom. ${ }^{[7]}$

On the whole, the term multi-modal discourse was used to emphasize the importance of considering semiotics such as image, music, gestures in analyzing language. With the development of technology, sound image, film, TV, computer and the Internet are undoubtedly the multi-modal discourse we produced. In brief, it is a trend to apply a multi-modal appreciation of meaning in language related work.

\section{Multi-modal discourse and Tujia folk song Long Chuan Diao English translation}

Folk song, the unique traditional culture, is the earliest one of produced language arts in the history of mankind. In China, folk song originated from the spring and autumn period in many different forms. While different parts of the folk song have different styles, some typical ones are Shanxi folk songs, Hunan folk songs, Xinjiang folk songs, involving different minorities. Tujia folk song, Long Chuan Diao, one of the 25 excellent folk songs in the world, widely sung in Hubei province, is adapted from Lichuan Lantern Song Zhong Gua Diao. As the important one of the folk dragon boat songs in Lichuan town, Long Chuan Diao depicts a vivid picture of a lively beautiful girl crossing the river by ferry when she goes home. It begins with an obvious long-distance greeting style of folk songs, the following short sentences with the rhythm of boating and the dance, which makes people feel fresh, lively, and humorous. The half-talk and half-singing dialogue reinforced the witty atmosphere. The last piece of music has both the image of the labour horn while rowing, presenting the image of singing the folk song again. It has rich features, artistic images, lifelike characters, cheerful melody and simple form, presenting rich life, creating a strong artistic appeal, and giving people dramatic aesthetic enjoyment. Under the comprehensive framework of Delu Zhang, this paper analyses the application of multimodal discourse in the translation of Tujia folk song Long Chuan Diao from four aspects: cultural level, context level, content level and expressive level.

\subsection{Cultural level}

Cultural level considers the cultural factors embodied in the folk songs of the Tujia nationality in western Hubei province. The translator needs to fully draw on the factors of Tujia culture in western Hubei province, without deviations in the understanding of the original text. This can be fully explained in terms of the translation of the title. The title Long Chuan Diao is translated into Dragon Boat song by means of literal translation. What's more, with the help of picture with dragon and boat, the translator successfully introduces the custom of Tujia dragon boat and reflects the scene characteristics of Tujia folk songs. Still, there is another example.

Chinese: Mei wa zi qu bai nian (nawei)! Mei wa (er) qu tan qin (na wei)

English: I am going for visits.

In Tujia culture, "Mei wa" is the term for the little sister in the narrow sense, broadly it can even refer to a girl who is older than himself but who looks very cute, also known as a younger sister which express people's love and indulgence. In this case, "Mei wa" here refers to the singer herself. Again, with a picture or the voice of a Tujia girl, it is a good way to translate it directly into "I" but not "She". At the same time, "Bai nian" and "Tan qin" both means "visit", and the corresponding English word "going for visits" is just enough with the help of some pictures.

\subsection{Context level}

Traditionally, context includes situational context and cultural context. In Tujia folk songs, their narrative context, scene context and Tujia culture have an impact on the translation.

The Tujia folk songs mainly include lots of love stories showing humanity origin. The love characterized culture of these folk songs is fully expressed and varies in styles, reflecting different love stages of Tujia people. Different love stages have different stresses, generally reflecting the human oriented character, that is, the Tujia people's strong desire for liberty of marriage, love as well as happiness. Look at the following example.

Chinese: Shao gong ni ba duo ban na, mei wa (er) wo shang le chuan.

English: You hold the rudder so tight, I look up into your eyes

Literally speaking, crossing the river by boat is one of the activities in daily life. However, in context here, the scene is the symbol of love of men and women. In the translation of Tujia folk songs, the translator paid attention to the influence of situational context, and adopted some appropriate techniques skilfully, translating the form of words "Shao gong" into "You", "shang le chuan" into "look up into your eyes". Besides the language, the use of picture of "Shao gong in the boat" makes the folk song effectively turn dull words into vivid love story, presenting a romantic story at the Qingjiang ferry scene. 


\subsection{Content level}

The content level consists of two parts, meaning level and formal level. In traditional translation studies, the concept of content and form is separated. But in the framework of comprehensive theory of multi-modal discourse analysis proposed by Zhang Delu, the content level includes two levels of form and meaning. The form level refers to different modalities, the representation of the state or symbolic system, and meaning level can be further analysed as ideational meaning, interpersonal meaning and textual meaning.

Here, the translation of Tujia folk songs involves the subject participation, so it must have certain subjectivity, which is indispensable to the understanding of the song. It is this understanding of translator that makes the song meaningful. This understanding is by no means an individual activity of human existence, and it makes the translator reveal the true meaning of the song. There is an example in the following.

Chinese: Jin na yin er suo yin na yin er suo,

Yang que jiao (wa yi wei zi yo, na ge yi ya wei zi yo). English: I see early birds, do you see the early birds?

They are singing what a pretty girl. Any one knows her?

In this level, the translator makes full use of the lyrics to enhance interaction through the question "do you see the early birds?", giving a lot of imagination to listeners. What's more, the increasing content of "what a pretty girl" is the foreshadowing of the love story between the younger sister and the boatman. The love song records faithfully the cultural heritages in Tujia Nationality in terms of artistic aesthetics.

\subsection{Expressive level}

Expression level can also be called media level, which is divided into language aspect and non-language aspect. Linguistic aspects include pure language and companion language. Under the background of folk song translation, pure language refers to the original lyrics and the translated text, the accompanying language refers to the font, size and stylistic layout, and the non-language aspect refers to the factors of video quality, picture, sound, media, and so on. One example can be shown in the following.

Chinese: Mei wa yao guo he wa? Na ge lai tui wo ma?

Wo lai tui ni ma!

English: A river lies there, how can I get there?

Let me ferry you there.

Language aspect is necessary to pay attention to. In the process of translating folk songs into English, the translator does not choose the question "who" in the expression, but the question "how", which shows the beauty of the rhythm. It is very difficult to be faithful to the original folk song and to conform to the rhythm of the music. If both of the originality and rhythm cannot be obtained, translators give up the former rather than undermine the rhyme of the song. At the same time, nonlanguage aspect can be presented by high mountains, flowing water, birds' words and flowers' fragrance. The song praises the love and life of the Tujia people in western Hubei province, makes the listener enter the hearts of the people of the world. Utilizing some ways such as male and female duet, lyrics, rhythm, pictures, action, the translator shows the meaning of the original text obviously.

\section{Significance of multi-modal discourse in the English translation of the Tujia folk songs in western Hubei province}

As a carrier of music, folk song is undoubtedly the most universal and influential literary style in contemporary cultural life. Its influence lies not only in its functions of entertainment, leisure, educational enlightenment, but also in its unique literary and artistic value. While the lyrics of Tujia folk songs are simple, popular, melodious, and their lyricism and folk emotion are very prominent, the unique language style of Tujia folk songs in western Hubei province is closely related to local characteristics. Today, China has entered a new and diverse cultural era. However, because of the impact of modern culture, many young people are keen on pop song, and folk song is facing a large area of extinction, especially minority songs. How to protect and inherit these folk songs is a matter of great urgency. In recent years, the protection of intangible cultural heritage has been gradually taken seriously.

\subsection{Understand Tujia culture}

A multi-modal discourse study on the English translation and dissemination of Tujia folk songs in western Hubei province can help to understand Tujia culture. The translation of folk songs into English is the artistic reproduction of song in people's daily life. By analysing the English translation and dissemination of Tujia folk songs in western Hubei province, this paper can improve people's understanding of Tujia folk song culture, and adapt to the needs of the construction and development of folk song under the modernization of our country. Only when the English translation is properly used, can culture be understood. The smell of mountains and flowers and plants, and the artistic charm can be truly developed, discussed, and spread to the world. These English translations have a high significance in spreading the local culture. Besides, it deepens people's understanding of Tujia people, promotes the development of local tourism, and facilitates the cultivation of local economy. The close combination of culture and social development has made greater contributions to the current Chinese culture "Going Out", the "Belt and Road" strategy and the development of socialist modernization.

\subsection{Guide the English translation of Tujia folk songs}

Folk songs are poems that can be sung. The translation is a re-elaboration of the contents of folk songs. It is a cross 
subject closely related to translation, literature and music. The existence of musical factors determines that the translator needs to consider whether the lyrics can match the melody of the song and finally make the song translation achieve the effect of singing. Lyrics are the most direct reappearance of folk songs, which fill up the contents of folk songs and endow them with profound cultural connotations and inner details. The translation of lyrics should not only conform to the rhythm of the original poem, but also match the music. Words, pictures and sounds are often used to enrich the musical image, deepen the expression of feelings, and make the lyric vivid and interesting in the traditional Chinese folk songs. Sometimes, some specific scenery, animal calls, and others are associated with folk songs in order to enrich imagination and deepen the theme. According to the multi-modal discourse theory, the translator interprets the folk songs into English, probes into the law of translation into English, and solves the problems in English translation, so as to guide the English folk song translators to understand and improve the level of English translation.

\subsection{Promote the interdisciplinary development}

Linguistics is a leading discipline in the humanities, both in terms of its theoretical structure and in terms of its precise task, which is the most advanced in the humanities and plays an important role in other disciplines. Tujia folk song itself is intoxicating language art, not only in various forms, beautiful and moving tunes, but also in simple and natural lyrics. Applying the theoretical methods and paradigm strategies of linguistics to the study of Tujia folk songs translation can enhance the scientific nature of the study and promote the interdisciplinary development. It is a topic of great significance.

\section{Conclusion}

Tujia folk songs are the simplest and most touching national treasures not only enriching people's spiritual life, but also encouraging people' kindness, diligence and wisdom. Multi-modal discourse and Tujia folk song translation are interdependent and complementary for folk song discourse contains many modal features, such as words, picture, rhythm and others. The translation of Tujia folk song discourse into English also has involved these multi-modal features, and the translator can make full use of them from the cultural level, the context level, content level and expressive level. Tujia folk songs construct discourse meaning mainly through visual mode and auditory mode. Thus, music and lyrics strengthen the prominent function of image. Multi-modal features also contribute to the translator's understanding, expression and interpretation in the process of translation, and bring the audience an indelible impression of shock. As Ruilan Cheng and Delu Zhang have said, there are some problems in the study of multi-modal discourse, such as the poor adaptability of multi-disciplines, and the method needs to be expanded. [8] Therefore, the construction of a comprehensive theoretical framework of multi-modal discourse analysis for English translation of folk songs will play an extremely important role in the translation and dissemination of Tujia folk songs in western Hubei province.

\section{Acknowledgment}

This paper is supported by The 2017 Youth Research Foundation Project in Hubei University for Nationalities: A study on the translation and dissemination of Tujia folk songs in western Hubei province from the perspective of multi-modal discourse analysis (MY2017Q012)

\section{References}

1. G. Kress, T. Van Leeuwen. Reading images: the grammar of visual design. (Routledge, London, 1996)

2. L. Zhanzi. Social semiotic analysis of multi-modal discourse. F.L.S, 5 (2003)

3. Z. Delu. A study on the comprehensive theoretical framework of multi-modal discourse analysis. C.F.L, 1 (2009)

4. F. Dezheng. Digital technology and multi-modal discourse analysis. J.B. J.U.S.T , 4 (2010)

5. C. Dan. Multi-modal discourse analysis of minority TV cultural programs. E. S.G.Z, 8 (2015)

6. L. Yanzhi. Subtitle translation of Anglo-American film and TV dramas from the perspective of multimodal discourse analysis. C.T.V, 12 (2016)

7. Z. Weiping. A study on the theoretical framework of multi-modal discourse analysis in flipping classroom. A.E.C, 8 (2017)

8. C. Ruilan, Z. Delu. The present situation, characteristics and development trend of multimodal discourse analysis in China: take the journal achievement as an example. C.F.L, 5 (2017) 